\title{
CONSTRUCTION OF LYAPUNOV FUNCTIONS ON THE DOMAIN OF ASYMPTOTIC NULLCONTROLLABILITY: THEORY
}

\author{
Fabio Camilli ${ }^{*}$ Lars Grüne ${ }^{* *}$ Fabian Wirth ${ }^{* *, 1}$ \\ * Sez. di Matematica per l'Ingegneria, Dip. di Matematica Pura e \\ Applicata, Università de l'Aquila, 67040 Monteluco di Roio (AQ), \\ Italy, camilli@ing.univaq.it \\ ** Mathematisches Institut, Fakultät für Mathematik und Physik, \\ Universität Bayreuth, 95440 Bayreuth, Germany, \\ lars.gruene@uni-bayreuth.de \\ *** Hamilton Institute, NUI Maynooth, Maynooth, Co. Kildare, \\ Ireland, Fabian.Wirth@may.ie
}

\begin{abstract}
In this paper we study the domain of asymptotic nullcontrollability of control systems. It is shown that this domain may be characterized by optimal control problems whose value function is the unique viscosity solution of a suitable first-order PDE. This value function turns out to be a control Lyapunov function. The approach generalizes Zubov's method.
\end{abstract}

Keywords: Asymptotic nullcontrollability, domain, Zubov's method, control Lyapunov function.

\section{INTRODUCTION}

In this paper we consider finite-dimensional nonlinear control systems that are asymptotically null controllable in a neighborhood of the origin and study the construction of control Lyapunov functions on that neighborhood. The relation between asymptotic null-controllability and the existence of control Lyapunov functions has attracted wide interest recently. In (Sontag, 1983) it is shown that (global) asymptotic nullcontrollability is equivalent to the existence of a control Lyapunov function using optimal control methods. A discussion of the literature control Lyapunov functions can be found in (Sontag, 1999).

Constructive approaches to control Lyapunov functions have received widespread attention most notably with the techniques known as backstep-

1 This work was supported by Science Foundation Ireland grant 00/PI.1/C067 ping and forwarding. In (Freeman and Kokotovic, 1996) it is shown that a smooth robust control Lyapunov functions is the solution of a suitable Hamilton-Jacobi-Isaacs equation making another connection of the field with optimal control. In a different approach in (Dubljevič and Kazantsis, 2002) Zubov's method is applied to single-input systems and control Lyapunov functions are obtained by truncation of series solutions. This approach resembles earlier works on approximate series solutions of Zubov's equation as e.g. (Kirin et al., 1982; Vannelli and Vidyasagar, 1985). The approach taken in the present paper uses ideas from (Camilli et al., 2001) where perturbed systems are studied and also further references on Zubov's method may be found.

We proceed as follows: In the ensuing Section 2 we define the class of systems under consideration and introduce the problem. In Section 3 we introduce the optimal control problem that characterizes the domain of asymptotic nullcon- 
trollability. In Section 4 we show that the value function of this optimal control problem is the unique viscosity solution of a generalization of Zubov's equation.

\section{NULL-CONTROLLABILITY}

We consider nonlinear control systems of the type

$$
\dot{x}(t)=f(x(t), u(t)),
$$

where $f: \mathbb{R}^{n} \times U \rightarrow \mathbb{R}$ is continuous, $U \subset \mathbb{R}^{m}$ is a closed set and the space of admissible control functions is given by

$$
u \in \mathcal{U}:=L^{\infty}([0, \infty), U) .
$$

Solutions corresponding to an initial value $x$ and a control $u \in \mathcal{U}$ at time $t$ are denoted by $\varphi(t, x, u)$. They are defined on a maximal positive interval of definition $\left[0, T_{\max }(x, u)\right)$, where we do not exclude the case that $T_{\max }(x, u)<\infty$. In the following the open ball of radius $r$ around a point $z \in \mathbb{R}^{p}$ is denoted by $B(z, r)$.

Uniqueness of solutions is a consequence of our further standard assumption on $f$, which are formulated using comparison functions. ${ }^{2}$

There exists $\gamma \in \mathcal{K}_{\infty}$ such that for any $R>0$ there is $C_{R}>0$ with

$\|f(x, u)-f(y, u)\| \leq C_{R}(1+\gamma(\|u\|))\|x-y\|$, for all $x, y$ with $\|x\|,\|y\| \leq R$,

(H1) $f(0,0)=0$,

There exists an open ball $B(0, r)$, a constant $\bar{u}>0$, and $\beta \in \mathcal{K} \mathcal{L}$ such that for (H2) any $x \in B(0, r)$ there exists $u_{x} \in \mathcal{U}$ with $\left\|u_{x}\right\|_{\infty} \leq \bar{u}$ and

$$
\left\|\varphi\left(t, x, u_{x}\right)\right\| \leq \beta(\|x\|, t) .
$$

Remark 1. The Lipschitz assumption (H0) is weaker than the following assumption:

For any $R>0$ there exists $C_{R}>0$ with

$$
\|f(x, u)-f(y, u)\| \leq C_{R}(1+\|u\|)\|x-y\|,
$$

for all $x, y$ with $\|x\|,\|y\| \leq R$,

which is used in (Soravia, 1999a; Soravia, 1999b), the results of which we will use later. In order to be able to use these results using (H0) we define an input transformation $R(u)=\gamma^{-1}(\|u\|) u /\|u\|$ and consider the vector field

$$
\hat{f}(x, u)=f(x, R(u))
$$

\footnotetext{
2 As usual we call a function $\alpha$ of class $\mathcal{K}_{\infty}$ if it is a homeomorphism of $[0, \infty)$, a continuous function $\beta$ in two real nonnegative arguments is called of class $\mathcal{K} \mathcal{L}$ if it is of class $\mathcal{K}_{\infty}$ in the first and decreasing to zero in the second argument.
}

with $u \in \widetilde{U}:=R^{-1}(U)$. This system satisfies

$$
\|\hat{f}(x, u)-\hat{f}(y, u)\| \leq C_{R}(1+\|u\|)\|x-y\| .
$$

Hence the results of (Soravia, 1999a; Soravia, $1999 b$ ) are applicable to our case.

Property (H2) is a local asymptotic controllability property. It is known (Sontag, 1998) that for any $\beta \in \mathcal{K} \mathcal{L}$ there exist two functions $\alpha_{1}, \alpha_{2} \in \mathcal{K}^{\infty}$ such that $\beta(r, t) \leq \alpha_{2}\left(\alpha_{1}(r) e^{-t}\right)$. For ease of presentation we use this upper bound.

We define the domain of null controllability by

$$
\begin{array}{r}
\mathcal{D}_{0}:=\left\{x \in \mathbb{R}^{n} \mid \text { there exists } u \in \mathcal{U}\right. \text { with } \\
\|\varphi(t, x, u)\| \rightarrow 0 \text { for } t \rightarrow \infty\} .
\end{array}
$$

Recall that a set $M$ is called viable, if $\forall x \in$ $M \exists u \in \mathcal{U}$ such that $\varphi(t, x, u) \in M$ for all $t \geq 0$. Using ideas similar to those in (Grüne and Wirth, 2000) the following can be shown.

Proposition 2. Assume (H0)-(H2). Then the following properties hold.

(i) $\operatorname{cl} B(0, r) \subset \mathcal{D}_{0}$,

(ii) the set $\mathcal{D}_{0}$ is open, connected and viable.

\section{OPTIMAL CONTROL CHARACTERIZATION OF $\mathcal{D}_{0}$}

In this section $\mathcal{D}_{0}$ is characterized via an optimal control problem and the continuity of the corresponding value function is shown. In order to set up the problem we need a running cost $g$ satisfying the following assumptions:

The function $g: \mathbb{R}^{n} \times U \rightarrow \mathbb{R}$ is continuous and satisfies $(\mathrm{H} 0)$ with the same $\gamma \in \mathcal{K}_{\infty}$

as $f$. Furthermore, for all $c>0$ we have

$$
\inf \{g(x, u) \mid\|x\| \geq c, u \in U\}=: g_{c}>0 \text {. }
$$

We need to ensure convergence of the integral cost that is introduced shortly for the "right" stabilizing solutions. To this end we assume that

$$
g(x, u) \leq C \alpha_{2}^{-1}(\|x\|),
$$

for all $(x, u) \in B(0, r) \times B(0, \bar{u})$.

$$
\begin{aligned}
& \quad g(x, u) \geq\|f(x, u)\|+\gamma(\|u\|), \\
& \text { whenever }\|x\| \geq 2 r \text { or }\|u\| \geq 2 \bar{u} .
\end{aligned}
$$

For $u$ such that $T_{\max }(x, u)=\infty$ we define the functional

$$
J(x, u):=\int_{0}^{\infty} g(\varphi(t, x, u), u(t)) d t,
$$

and we set $J(x, u):=\infty$, if $T_{\max }(x, u)<\infty$. We define furthermore the (extended real valued) optimal value function

$$
V(x):=\inf _{u \in \mathcal{U}} J(x, u), \quad x \in \mathbb{R}^{n},
$$


and the function $v(x):=1-e^{-V(x)}, \quad x \in \mathbb{R}^{n}$.

Note that both $V$ and $v$ satisfy appropriate dynamic programming principles, i.e., for each $T>0$ we have

$V(x)=\inf _{u \in \mathcal{U}}\left\{\int_{0}^{T} g(\varphi(t, x, u), u(t)) d t+V(\varphi(T))\right\}$

and

$$
v(x)=\inf _{u \in \mathcal{U}}\{1+G(x, T, u)(v(\varphi(T, x, u))-1)\},
$$

where

$$
G(x, T, u):=\exp \left(-\int_{0}^{T} g(\varphi(t, x, u), u(t)) d t\right) .
$$

We now investigate the properties of $V$ and $v$. For this purpose using the function $\gamma$ from (H0) we define for $u \in \mathcal{U}$

$$
\|u\|_{\gamma, T}:=\int_{0}^{T} \gamma(\|u(t)\|) d t .
$$

Lemma 3. Let $T>0$. If $x \in \mathbb{R}^{n}$ and $u \in \mathcal{U}$ are such that $\|\varphi(t, x, u)\| \geq 2 r, t \in[0, T]$ or $\|u(t)\| \geq 2 \bar{u}$ a.e. $t \in[0, T]$, then

$\int_{0}^{T} g(\varphi(t, x, u), u(t)) d t \geq\|\varphi(T, x, u)-x\|+\|u\|_{\gamma, T}$

Proposition 4. Assume (H0)-(H4), then

(i) $V(x)<\infty$ and $v(x)<1$ if and only if $x \in \mathcal{D}_{0}$,

(ii) if in addition (H5) holds, then $V(x)=0 \Leftrightarrow$ $x=0$ and $v(x)=0 \Leftrightarrow x=0$.

Proof. We show the statements for $V$; where (i) may be shown using ideas as in (Camilli et al., 2001).

(ii) It is clear that $V(0)=0$, so let $x \neq 0$. Assume to the contrary that there is a sequence $\left\{u_{k}\right\} \subset \mathcal{U}$ such that $J\left(x, u_{k}\right) \rightarrow 0$. Let $c:=\|x\| / 2$ and denote

$$
t_{k}:=\inf \left\{t \geq 0 \mid\left\|\varphi\left(t, x, u_{k}\right)\right\| \leq c\right\} .
$$

By (H3) we have for all $k$ that $J\left(x, u_{k}\right) \geq$ $\int_{0}^{t_{k}} g\left(\varphi\left(s, x, u_{k}\right), u_{k}(s)\right) d s \geq t_{k} g_{c}$ which implies that $t_{k} \rightarrow 0$. Now $\|f\|$ is bounded on $W:=$ $B(0,2 r) \times B(0,2 \bar{u})$ by the constant $C:=C_{2 r}(1+$ $\gamma(2 \bar{u})) 2 r$. Denote by $E(k)$ the set

$$
\left\{t \in\left[0, t_{k}\right] \mid\left(\varphi\left(t, x, u_{k}\right), u_{k}(t)\right) \in W\right\},
$$

which is well defined up to a set of measure zero. Then

$$
\int_{E(k)}\left\|f\left(\varphi\left(t, x, u_{k}\right), u_{k}(t)\right)\right\| d t \leq t_{k} C .
$$

On the other hand we have for all $k$ that

$\int_{0}^{t_{k}}\left\|f\left(\varphi\left(t, x, u_{k}\right), u_{k}(t)\right)\right\| d t \geq\left\|x-\varphi\left(t_{k}, x, u_{k}\right)\right\|$.

Using (H5) this implies that

$$
J\left(x, u_{k}\right) \geq \int_{\left[0, t_{k}\right] \backslash E(k)} g\left(\varphi\left(s, x, u_{k}\right), u_{k}(s)\right) d s
$$

$\geq \int_{\left[0, t_{k}\right] \backslash E(k)}\left\|f\left(\varphi\left(s, x, u_{k}\right), u_{k}(s)\right)\right\| d s \geq c-t_{k} C$. As $t_{k} \rightarrow 0$ this contradicts $J\left(x, u_{k}\right) \rightarrow 0$.

We start our investigation by proving continuity properties for the trajectories of (1). The following is a consequence of Gronwall's lemma.

Lemma 5. Assume (H0) and let $T>0$ and $R>0$ be arbitrary constants. Then for all $x, y \in \mathbb{R}^{n}$ and all $u \in \mathcal{U}$ satisfying

$\|\varphi(t, x, u)\| \leq R, \quad\|\varphi(t, y, u)\| \leq R, \quad \forall t \in[0, T]$ we have

$$
\|\varphi(t, x, u)-\varphi(t, y, u)\| \leq e^{C_{R}\left(\|u\|_{\gamma, t}+t\right)}\|x-y\|,
$$

for all $t \in[0, T]$.

Now we can prove the desired continuity result.

Proposition 6. Assume (H0)-(H5), then $V$ is continuous on $\mathcal{D}_{0}$ and $v$ is continuous on $\mathbb{R}^{n}$.

Proof. We show the continuity of $V$ on $\mathcal{D}_{0}$, then the statement for $v$ follows immediately from its definition and Proposition 7. The proof is performed in several steps. First note that we have $V(x) \leq C \alpha_{1}(\|x\|), \quad$ for $x \in B\left(0, \alpha_{1}^{-1} \circ \alpha_{2}^{-1}(r)\right)$.

Using a concatenation argument this may be used to show that for $x_{0}$ in $\mathcal{D}_{0}$ there is a $\delta>0$ such that $\sup _{x \in B\left(x_{0}, \delta\right)} V(x)=: B_{V}$ is finite.

(i) (Bounds on $\varepsilon$-optimal controls and trajectories)

For any $x \in B\left(x_{0}, \delta\right)$ and any $\varepsilon \in(0,1]$ we pick $u_{x, \varepsilon} \in \mathcal{U}$ such that

$$
J\left(x, u_{x, \varepsilon}\right) \leq V(x)+\varepsilon .
$$

We claim that for any $\varepsilon>0$ the set

$$
K_{\varepsilon}:=\left\{\varphi\left(t, x, u_{x, \varepsilon}\right) \mid t \geq 0, x \in B\left(x_{0}, \delta\right)\right\}
$$

and the sets

$$
\left\{\left\|u_{x, \varepsilon}\right\|_{\gamma, T} \mid x \in B\left(x_{0}, \delta\right)\right\}, \quad T>0
$$

are bounded. If the first set were unbounded then there would be an $x \in B\left(x_{0}, \delta\right)$ and $t_{1}>0$ such that $\left\|\varphi\left(t_{1}, x, u_{x, \varepsilon}\right)\right\| \geq V(x)+2 \varepsilon+2 r$. If $t_{2}>t_{1}$ is the first time at which $\left\|\varphi\left(t_{1}, x, u_{x, \varepsilon}\right)\right\|=2 r$ again, then we obtain using Lemma 3 that

$$
J\left(x, u_{x, \varepsilon}\right) \geq V(x)+2 \varepsilon,
$$

a contradiction. The second claim is brought to a contradiction using Lemma 3 in a similar manner.

(ii) (Continuity of trajectories)

We denote by $R_{\varepsilon}$ an upper bound on the set $K_{\varepsilon}$. By Lemma 5 we can conclude that for $x, y \in$ $B\left(x_{0}, \delta\right)$ and all $t \geq 0$ such that

$$
\|x-y\| \leq R_{\varepsilon} \exp \left(-C_{2 R_{\varepsilon}}\left(\left\|u_{x, \epsilon}\right\|_{\gamma, t}+t\right)\right)
$$


we have

$$
\begin{array}{r}
\left\|\varphi\left(t, x, u_{x, \epsilon}\right)-\varphi\left(t, y, u_{x, \epsilon}\right)\right\| \\
\leq \exp \left(C_{2 R_{\varepsilon}}\left(\left\|u_{x, \varepsilon}\right\|_{\gamma, t}+t\right)\right)\|x-y\| .
\end{array}
$$

\section{(iii) (Continuity of $V$ )}

It suffices to show continuity of $V$ on $B\left(x_{0}, \delta\right)$. So pick $0<\varepsilon<\alpha_{2}^{-1}(r) C$. By (H3) and the finiteness of $J\left(x, u_{x, \varepsilon}\right)$ we may assume that the controls $u_{x, \varepsilon}$ are chosen in such a way that there exists $T_{\epsilon}>0$ (depending on $B_{V}$ ) such that

$$
\varphi\left(t, x, u_{x, \varepsilon}\right) \in B\left(0, \alpha_{1}^{-1}(\varepsilon / C) / 2\right),
$$

for all $t \geq T_{\epsilon}, x \in B(0, \delta)$. Denote

$$
\tilde{C}:=\exp \left(-C_{2 R_{\varepsilon}}\left(\max _{z \in B\left(x_{0}, \delta\right)}\left\|u_{z, \epsilon}\right\|_{\gamma, T_{\varepsilon}}+T_{\varepsilon}\right)\right),
$$

and note that the right hand side is finite by (i). Choose two points $x, y \in B\left(x_{0}, \delta\right)$ such that

$$
\|x-y\| \leq R_{\varepsilon} \tilde{C} .
$$

Without loss of generality assume $V(y) \geq V(x)$. Abbreviating $u:=u_{x, \varepsilon}, T:=T_{\varepsilon}$ we obtain

$$
\begin{aligned}
& |V(y)-V(x)|=V(y)-V(x) \\
& \leq \int_{0}^{T}|g(\varphi(t, y, u), u(t))-g(\varphi(t, x, u), u(t))| d t \\
& \quad+V(\varphi(T, y, u))+\varepsilon .
\end{aligned}
$$

Using (H3) and (5) we continue

$$
\begin{aligned}
\leq & \int_{0}^{T} C_{2 R_{\epsilon}}(1+\gamma(\|u(t)\|)) \widetilde{C}\|x-y\| d t \\
& +V(\varphi(T, y, u))+\varepsilon
\end{aligned}
$$

and we obtain

$$
\leq C_{2 R_{\varepsilon}}\left(T+\|u\|_{\gamma, T}\right) \widetilde{C}\|x-y\|+2 \varepsilon,
$$

provided $\|y-x\| \leq \alpha_{1}^{-1}(\varepsilon / C) /(2 \widetilde{C})$. It is now easy to show the assertion.

The next proposition concerns the behavior of $V(x)$ near the boundary of $\mathcal{D}_{0}$ or at $\infty$.

Proposition 7. Assume (H0)-(H5). Then for any sequence $x_{n}$ which satisfies $\operatorname{dist}\left(x_{n}, \partial \mathcal{D}_{0}\right) \rightarrow 0$ or $\left\|x_{n}\right\| \rightarrow \infty$ we have $V\left(x_{n}\right) \rightarrow \infty$ and $v\left(x_{n}\right) \rightarrow 1$.

Proof. This follows using similar arguments to the ones to the proof of Proposition 6 .

\section{ZUBOV'S METHOD}

The aim of this section is to characterize the functions $V$ and $v$ in the previous section as (the unique) viscosity solutions of the equations

$$
\sup _{u \in U}\{-D V(x) f(x, u)-g(x, u)\}=0
$$

and

$$
\sup _{u \in U}\{-D v(x) f(x, u)-(1-v(x)) g(x, u)\}=0,
$$

respectively (for the definition of viscosity solution we refer to (Bardi and Capuzzo-Dolcetta, 1997)). Note that it follows from these characterizations that $v$ is a control Lyapunov function on $\mathcal{D}_{0}$ in the usual sense, (Sontag, 1999). In fact, a small calculation shows that $v$ is a viscosity supersolution on $\mathcal{D}_{0}$ of

$$
\max _{u \in U}-D v(x) f(x, u) \geq W(x) g_{\|x\|},
$$

where $0<W(x):=1-v(x)$ for $x \in \mathcal{D}_{0} \backslash\{0\}$ and $g_{\|x\|}$ denotes the constant from (H3) for $c=\|x\|$.

In this section we drop the assumption (H5) and replace it by assumptions relating the growth of $f$ and $g$. To this end we define the functions

$$
\tilde{f}(x, u)=\frac{f(x, u)}{1+\|f(x, u)\|}, \tilde{g}(x, u)=\frac{g(x, u)}{1+\|f(x, u)\|} .
$$

Remark 8. The effect of the rescaling described above can be described as follows. Let $x \in \mathbb{R}^{n}, u \in$ $\mathcal{U}$ be given. Now introduce a new time variable $\tau$ through

$$
\frac{d t(\tau)}{d \tau}=\frac{1}{1+\|f(\phi(t(\tau), x, u), u(t(\tau)))\|}, \text { a.e., }
$$

and a control $\tilde{u}(\tau):=u(t(\tau))$, a.e. Then $\psi(\tau):=$ $\phi(t(\tau), x, u)$ satisfies the differential equation

$$
\frac{d \psi(\tau)}{d \tau}=\tilde{f}(\psi(\tau), \tilde{u}(\tau)) .
$$

So if we consider the system

$$
\dot{x}(t)=\tilde{f}(x(t), u(t)),
$$

it is easy to see that if $f$ satisfies (H0), (H1) and (H2) then also $\tilde{f}$ satisfies these properties for suitably adjusted functions $\tilde{\gamma}$ and $\tilde{\beta}$. Also $\mathcal{D}_{0}$ is the same for $f$ and $\tilde{f}$.

Consider now the optimal control problem for system (8) given by the running cost $\tilde{g}$. Then using standard transformation of integral formulas it is also easy to see that $\tilde{J}(x, \tilde{u})=J(x, u)$, where $\tilde{J}$ is defined using $\tilde{g}$ in the spirit of (3). In particular, this implies that the value functions $v$ and $V$ coincide for the optimal control problems defined through (1) and (3), and the rescaled version.

Note also, that $\tilde{g}$ clearly satisfies (H4) with a modified decay $\tilde{\alpha}_{2}$, as well as the first statement of (H3), whereas the lower bound $g_{c}$ need not exist. This is no problem, however, as $g_{c}$ is only used to ensure that trajectories not converging to 0 result in the value $\infty$ for the original optimal control problem. As the value associated with a trajectory does not change, this property is preserved under our transformation.

Now we introduce the following assumption, which implies (H5). 
(H6)

$$
\begin{aligned}
& g(x, u) \geq\|f(x, u)\|+\gamma(\|u\|)(1+\|f(x, u)\|), \\
& \text { whenever }\|x\| \geq 2 r \text { or }\|u\| \geq 2 \bar{u},
\end{aligned}
$$

To (6) and (7) we associate the Hamiltonians

$$
H_{V}(x, p)=\sup _{u \in U}\{-f(x, u) p-g(x, u)\},
$$

and

$$
H_{v}(x, r, p)=\sup _{u \in U}\{-f(x, u) p-(1-r) g(x, u)\} .
$$

Given (H6) (and assuming $r<1$ for $H_{v}$ ) the Hamiltonians $H_{V}$ and $H_{v}$ are locally Lipschitz continuous with respect to their arguments. Also, recalling that $V$ is locally bounded in $\mathcal{D}_{0}$ and $v$ is bounded in $\mathbb{R}^{n}$, our first result follows from a standard application of the dynamic programming principles satisfied by $v$ and $V$, see (Bardi and Capuzzo-Dolcetta, 1997).

Proposition 9. Assume (H0) - (H6), then $V$ is a viscosity solution of (6) in $\mathcal{D}_{0}$ and $v$ is a viscosity solution of (7) in $\mathbb{R}^{n}$.

The following is the main theorem of our paper.

Theorem 10. Assume that (H0)-(H6) hold. Then

(i) $v$ is the unique bounded continuous viscosity solution $v$ of (7) vanishing at the origin,

(ii) $\left(\mathcal{D}_{0}, V\right)$ is the unique couple of an open set containing the origin and a locally bounded, continuous viscosity solution of (6) in $\mathcal{O}$ with $V(0)=0$ and $V(x) \rightarrow+\infty$ for $x \rightarrow \partial \mathcal{D}_{0}$.

In the proof we encounter two difficulties: the unbounded dependence of the coefficients on the control variable and the vanishing of the cost $g$ at the origin. To solve the first problem we introduce two rescaled equations which share with (6) and (7) the same set of sub- and supersolutions.

Lemma 11. Assume (H0)-(H6) and consider the equations

$$
\sup _{u \in U}\{-D \widetilde{V}(x) \tilde{f}(x, u)-\tilde{g}(x, u)\}=0
$$

and

$$
\sup _{u \in U}\{-D \tilde{v}(x) \tilde{f}(x, u)-(1-\tilde{v}(x)) \tilde{g}(x, u)\}=0,
$$

for the functions $\tilde{f}$ and $\tilde{g}$. Then any viscosity subsolution (supersolution) of (6) is a viscosity subsolution (supersolution) for (9) and vice versa. The same assertions hold for (10) and (7).

Proof. We prove the lemma for subsolutions of (6) and (9), the assertions supersolutions and for (7) and (10) follow by very similar arguments.

If $V^{-}$is a viscosity subsolution of (6), then for any supergradient $p$ of $V^{-}$in $x$ we have that

$$
\sup _{u \in U}\{-f(x, u) p-g(x, u)\} \leq 0 .
$$

This implies

$$
-f(x, u) p-g(x, u) \leq 0, \text { for all } u \in U
$$

and since $1+\|f(x, u)\|$ is positive, this implies

$$
-\tilde{f}(x, u) p-\tilde{g}(x, u) \leq 0, \text { for all } u \in U,
$$

which in turn implies

$$
\sup _{u \in U}\{-\tilde{f}(x, u) p-\tilde{g}(x, u)\} \leq 0,
$$

hence $V^{-}$is a viscosity supersolution of (9).

The converse direction follows by the same argument, since again we multiply by a positive factor, now $1+\|f(x, u)\|$.

The following corollary is a simple consequence of this lemma.

Corollary 12. Assume (H0)-(H6), then

(i) Any viscosity solution of (6) is a viscosity solution of (9) in $\mathcal{D}_{0}$ and vice versa.

(i) Any viscosity solution of (7) is a viscosity solution of (10) and vice versa.

In order to prove a uniqueness result for (9) and (10), we use a control theoretic argument and some optimality principles introduced in (Soravia, 1999a; Soravia, 1999b), as stated in the following lemma.

Lemma 13. Assume (H0) and (H3) and denote by $\tilde{\varphi}(t, x, u)$ the solution of (8). Define

$$
\tilde{G}(x, t, u):=\exp \left(-\int_{0}^{t} \tilde{g}(\tilde{\varphi}(\tau, x, u), u(\tau)) d \tau\right) .
$$

Then

(i) Any upper semicontinuous viscosity subsolution $w^{-}$of (10) is pointwise upper bounded by

$$
\inf _{u \in \mathcal{U}} \inf _{t \in[0, T]}\left\{1+\tilde{G}(x, t, u)\left(w^{-}(\tilde{\varphi}(t, x, u))-1\right)\right\} .
$$

for each $T>0$.

(ii) Consider a continuous viscosity supersolution $w^{+}$of (10) and let $\Omega \subset \mathbb{R}^{n}$ be an open and bounded set with $\sup _{x \in \Omega} w^{+}(x)<1$. Consider the first exit time from $\Omega$ given by

$$
T_{e x}(x, u, \Omega)=\min \left\{t \geq 0 \mid \varphi\left(t, x_{0}, u\right) \notin \Omega\right\} .
$$

Then $w^{+}$is a pointwise upper bound for

$$
\inf _{u \in \mathcal{U}} \sup \left\{1+\tilde{G}(x, t, u)\left(w^{+}(\tilde{\varphi}(t, x, u))-1\right)\right\},
$$

where the second supremum is taken over $t \in$ $\left[0, T_{e x}(x, u, \Omega)\right]$.

Using these inequalities we can now prove our uniqueness results. 
Proof. of Theorem 10 We prove only (i), since the proof of assertion (ii) is similar. Note that by Remark 8 the functions $v$ and $V$ can be taken to be defined through (8) and the running cost $\tilde{g}$. In the following we work with this representation. Again by $\tilde{\varphi}(t, x, u)$ we denote the solutions of (8). Claim 1: If $w^{-}$is a bounded u.s.c. subsolution of (10) on $\mathbb{R}^{n}$ with $w^{-}(0) \leq 0$, then $w^{-} \leq v$.

By the upper semicontinuity of $w^{-}$and $w^{-}(0) \leq 0$ we obtain that for every $\varepsilon>0$ there exists a $\delta>0$ with $w^{-}(x) \leq \varepsilon$ for all $x \in \mathbb{R}^{n}$ with $\|x\| \leq \delta$. Now we distinguish two cases:

(i) $x_{0} \in \mathcal{D}_{0}$ : We choose $u^{*} \in \mathcal{U}$ such that $v\left(x_{0}\right)+$ $\varepsilon>\tilde{J}\left(x_{0}, u^{*}\right)=1-\tilde{G}\left(x_{0}, \infty, u^{*}\right)$. In particular, using (H3) and the final statement of Remark 8 this implies that there exists a sequence $t_{k} \rightarrow \infty$ such that $\tilde{\varphi}\left(t_{k}, x_{0}, u^{*}\right) \rightarrow 0$ as $k \rightarrow \infty$. Thus it follows from the lower optimality principle (11) and the definition of $v$ that

$$
\begin{array}{r}
w^{-}\left(x_{0}\right) \leq \\
\limsup _{k \rightarrow \infty} 1+\tilde{G}\left(x_{0}, t_{k}, u^{*}\right)\left(w^{-}\left(\tilde{\varphi}\left(t_{k}, x_{0}, u^{*}\right)\right)-1\right) \\
\leq 1+\tilde{G}\left(x_{0}, \infty, u^{*}\right)(\varepsilon-1) \leq v\left(x_{0}\right)+2 \varepsilon
\end{array}
$$

which shows the claim as $\varepsilon>0$ was arbitrary.

(ii) $x_{0} \notin \mathcal{D}_{0}$ : In this case by Proposition 4 it is sufficient to show that $w^{-}\left(x_{0}\right) \leq 1$. Let $M$ be a bound on $\left|w^{-}\right|$.

In the following we use $t$ as the variable for (1) and $\tau$ for the time-variable of (8) as in Remark 4.1. Since $\tilde{\varphi}\left(\tau, x_{0}, u\right) \notin B(0, r)$ for all $\tilde{u} \in \mathcal{U}$ and all $\tau \geq 0$ by (H3) and Remark 8 we have

$$
\int_{0}^{\tau} \tilde{g}\left(\tilde{\varphi}\left(s, x_{0}, u\right), \tilde{u}(s)\right) d s \geq g_{r} t(\tau)
$$

for the constant $g_{r}>0$ from (H3). Therefore $\tilde{G}\left(x_{0}, \tau, \tilde{u}\right) \leq \exp \left(-\tilde{g}_{r} t(\tau)\right)$ for all $\tau \geq 0, \tilde{u} \in \mathcal{U}$. Hence

$$
\begin{array}{r}
1+\tilde{G}\left(x_{0}, \tau, \tilde{u}\right)\left(w^{-}\left(\tilde{\varphi}\left(\tau, x_{0}, \tilde{u}\right)\right)-1\right) \\
\leq 1+\exp \left(-g_{r} t(\tau)\right)(M+1)
\end{array}
$$

for all $\tilde{u} \in \mathcal{U}$ and the result follows by (11) as the right hand side tends to 1 for $\tau \rightarrow \infty$.

Therefore Claim 1 is proved. Consider now

Claim 2: Let $w^{+}$be a bounded lower semicontinuous supersolution of (7) on $\mathbb{R}^{n}$ with $w^{+}(0) \geq 0$. Then $w^{+} b e \geq v$. The proof is omitted for reasons of space. The combination of Claim 1 and 2 yields the proof.

\section{CONCLUSION}

We have generalized Zubov's method to the problem of calculating domains of null-controllability and maximal control Lyapunov functions. A discussion of the numerical merits of the methods can be found in (Camilli et al., 2004).

\section{REFERENCES}

Bardi, M. and I. Capuzzo-Dolcetta (1997). Optimal control and viscosity solutions of Hamilton-Jacobi-Bellman equations. Systems \& Control: Foundations \& Applications. Birkhäuser. Boston, MA.

Camilli, F., L. Grüne and F. Wirth (2001). A generalization of Zubov's method to perturbed systems. SIAM J. Control Optim. 40(2), 496515.

Camilli, F., L. Grüne and F. Wirth (2004). Construction of Lyapunov functions on the domain of asymptotic nullcontrollability: numerics. In: these proceedings. Stuttgart.

Dubljevič, S. and N. Kazantsis (2002). A new Lyapunov design approach for nonlinear systems based on Zubov's method. Automatica 38, 1999-2007.

Freeman, R. A. and P. V. Kokotovic (1996). Inverse optimality in robust stabilization. SIAM J. Control Optim. 34(4), 1365-1391.

Grüne, Lars and Fabian Wirth (2000). Computing control Lyapunov functions via a Zubov type algorithm. In: Proc. 39th Conference on Decision and Control. Sydney, AUS. pp. 21292134.

Kirin, N. E., R. A. Nelepin and V. N. Bă̌daev (1982). Construction of the domain of attraction by Zubov's method. Differ. Equations $\mathbf{1 7}(8), 871-880$.

Sontag, E. D. (1983). A Lyapunov-like characterization of asymptotic controllability. SIAM J. Control Optim. 21(3), 462-471.

Sontag, E. D. (1998). Comments on integral variants of ISS. Systems Control Lett. 34(12), 93-100.

Sontag, E. D. (1999). Stability and stabilization: discontinuities and the effect of disturbances. In: Nonlinear analysis, differential equations and control (Montreal, QC, 1998). Vol. 528 of NATO Sci. Ser. C Math. Phys. Sci.. pp. 551598. Kluwer Acad. Publ.. Dordrecht.

Soravia, P. (1999a). Optimality principles and representation formulas for viscosity solutions of Hamilton-Jacobi equations. I. Equations of unbounded and degenerate control problems without uniqueness. Adv. Differential Equations 4(2), 275-296.

Soravia, P. (1999b). Optimality principles and representation formulas for viscosity solutions of Hamilton-Jacobi equations. II. Equations of control problems with state constraints. Differential Integral Equations 12(2), 275293.

Vannelli, A. and M. Vidyasagar (1985). Maximal Lyapunov functions and domains of attraction for autonomous nonlinear systems. $A u$ tomatica J. IFAC 21(1), 69-80. 